\title{
The role of rotation on Petersen diagrams
}

\section{The influence of near-degeneracy}

\author{
J. C. Suárez ${ }^{1,2, \star}$, R. Garrido ${ }^{1}$, and A. Moya ${ }^{1}$ \\ 1 Instituto de Astrofísica de Andalucía (CSIC), CP3004 Granada, Spain \\ e-mail: jcsuarez@iaa.es \\ 2 Observatoire de Paris, LESIA, UMR 8109, Meudon, France
}

Received 13 April 2007 / Accepted 7 August 2007

\begin{abstract}
Aims. In the present work, the effect of near-degeneracy on rotational Petersen diagrams (RPD) is analysed. Methods. Seismic models are computed considering rotation effects on both equilibrium models and adiabatic oscillation frequencies (including second-order near-degeneracy effects). Contamination of coupled modes and coupling strength on the first radial modes are studied in detail.

Results. Analysis of relative intrinsic amplitudes of near-degenerate modes reveals that the identity of the fundamental radial mode and its coupled quadrupole pair are almost unaltered once near-degeneracy effects are considered. However, for the first overtone, a mixed radial/quadrupole identity is always predicted. The effect of near-degeneracy on the oscillation frequencies becomes critical for rotational velocities larger than $15-20 \mathrm{~km} \mathrm{~s}^{-1}$, for which large wriggles in the evolution of the period ratios are obtained (up $10^{-2}$ ). Such wriggles imply uncertainties, in terms of metallicity determinations using RPD, reaching up to 0.50 dex, which can be critical for Pop. I HADS (High Amplitude $\delta$ Scuti stars). In terms of mass determinations, uncertainties reaching up to $0.5 M_{\odot}$ are predicted. The location of such wriggles is found to be independent of metallicity and rotational velocity, and governed mainly by the avoidedcrossing phenomenon.

Conclusions. Near-degeneracy affects significantly the $\Pi_{1 / 0}(\Omega)$ period ratios even for relatively low rotational velocities, and that can be critical when accurate determinations of mass and metallicity are required. Nevertheless, analysis of near-degeneracy effects provides some clues for the identification of the fundamental radial mode, the first overtone, and their corresponding quadrupole coupled pairs. This can be especially useful when additional information on mode identification and/or metallicity is available, for example from multicolour photometry and/or spectroscopy, not only for accurate diagnostics on metallicity and mass, but also because it is possible to constrain, to some extent, the rotational velocity of the star (and thereby its inclination angle).
\end{abstract}

Key words. stars: variables: $\delta$ Sct - stars: rotation - stars: oscillations - stars: fundamental parameters - stars: interiors

\section{Introduction}

As explained in several papers (Dziembowski \& Goode 1992; Soufi et al. 1998; Suárez et al. 2006b), the effects of rotation on oscillations (near-degeneracy included) cannot be neglected for asteroseismic studies even for relatively slow-rotating stars. Near-degeneracy affects the small separations since it occurs for close modes (under certain selection rules, $\Delta \ell=0, \pm 2$, and $\Delta m=0$ ). The effect of near-degeneracy on the oscillation modes is two-fold: it changes the oscillation frequencies (through the coupling strength) and the mode character (mode contamination). While the former effect is proportional to the rotation rate, the mode contamination is nearly independent of the rotation rate and strongly dependent on the mode degree $\ell$.

In the present paper we investigate the impact of including near-degeneracy in the asteroseismic diagnostics using rotational Petersen diagrams (hereafter RPD) for the range of mass, metallicity and rotational velocities studied in (Suárez et al. 2006a, from now on Paper I). In Paper I, the effect of rotation on period ratios of radial modes was explored focusing on double-mode pulsators. Detailed seismic models were computed considering rotation effects on both equilibrium models and on adiabatic oscillation frequencies. In particular, for $1.8 M_{\odot}$

* Associate researcher at institute (2). stellar models, period ratios were calculated for different rotational velocities (RPD) and metallicities and then compared with standard non-rotating Petersen diagrams (PD). We showed that the difference in period ratios increases with the rotational velocity for a given metallicity. Such differences in period ratios were found equivalent to differences in the metallicity of models up to 0.30 dex. The detailed investigation of this confusion scenario is therefore particularly relevant when accurate metallicity and/or mass determinations are required. As stated in Paper I, such ambiguity may still be increased when including different stellar masses, rotational velocities and metallicities, etc. Important effects are expected to be found when near-degeneracy is taken into account (Pamyatnykh 2003). In that work, the behaviour of $\Pi_{1 / 0}(\Omega)$ when near-degeneracy effects are included were studied for a typical $1.8 M_{\odot} \delta$ Scuti stellar model with a surface rotational velocity of $100 \mathrm{~km} \mathrm{~s}^{-1}$. He showed that very large and non-regular perturbations of such ratios are expected to occur.

The paper is structured as follows: General description of the modelling, focusing on how rotation is taken into account is given in Sect. 2; in Sect. 3 the effect of near-degeneracy on the oscillation frequencies, as well as the coupled mode contamination, is analysed for the fundamental radial mode and the first overtone; in Sect. 4, the impact of near-degeneracy on the RPD 
diagnostic diagrams is discussed; finally, conclusions are given in Sect. 5.

\section{The modelling}

Proceeding as in Paper I, equilibrium models are computed with the evolutionary code CESAM (Morel 1997), for which a first-order effect of rotation is taken into account in equilibrium equations. Uniform rotation and global conservation of the total angular momentum is assumed. We recall that this first-order effects of rotation are considered by subtracting the spherically averaged contribution of the centrifugal acceleration to the gravity of the model. This spherically averaged component of the centrifugal acceleration does not change the order of the hydrostatic equilibrium equations. Such models are the so-called "pseudorotating" models (see Soufi et al. 1998; Suárez et al. 2006b). Although the non-spherical components of the centrifugal acceleration are not considered, they are included as a perturbation in the oscillation frequencies computation.

A grid of equilibrium models is built, similar to the one used in Paper I, which is composed of models with masses ranging from 1.80 to $2 M_{\odot}$, with seven different metallicities: $[\mathrm{Fe} / \mathrm{H}]=0,-0.1,-0.2,-0.30,-0.35,-0.50$ and $-1.00 \mathrm{dex}$. The initial (ZAMS) rotational velocity of models is restricted to $\Omega_{\mathrm{i}}=15-50 \mathrm{~km} \mathrm{~s}^{-1}$. We recall that, due to the global conservation of the total angular momentum during the evolution, the rotational velocity of the models can decrease up to $0.75 \Omega_{\mathrm{i}}$ at the end of the main sequence (close to the terminal age main sequence, TAMS). A mixing-length parameter of $\alpha_{\mathrm{ML}}=1.80$ and an overshooting parameter of $d_{\mathrm{OV}}=0.2$ are assumed.

Computation of the oscillation spectra is carried out using the oscillation code FILOU (Tran Minh \& Léon 1995; Suárez 2002), which is based on a perturbative analysis and provides adiabatic oscillations, corrected for the effects of rotation up to the second order (centrifugal and Coriolis forces), including near-degeneracy effects.

\section{Near-degenerate oscillation frequencies}

In order to analyse the role of rotation on $\Pi_{1 / 0}(\Omega)$ period ratios, the complete second-order (in $v_{\Omega}$, the rotation rate) formalism given in (Suárez et al. 2006b, hereafter SGM06) is considered. The near-degeneracy effect can be divided into two: the effect on the oscillation frequencies, and the mode contamination, i.e. the weight of the original individual spherical harmonics describing the oscillation mode in the resulting coupled mode. Both aspects are theoretically analysed in Lochard et al. (2007) for solar-like, main-sequence stars. In that work, which is based on the developments given in SGM06, the authors use a method to numerically remove such disturbing effects on the small separations in order to analyse the echelle diagrams for rotating stars free of rotational effects from the observed frequencies. In the present work, we apply the developments given in those works to the specific case of the $\Pi_{1 / 0}(\Omega)$ period ratios. To do so, we selected from the model grid described in Sect. 2, eight models (see Table 1 ) with similar evolutionary stage $(1.3 \mathrm{Gyr})$, mean density, effective temperature, and with two different initial rotational velocities: $\Omega_{\mathrm{i}}=25$, and $50 \mathrm{~km} \mathrm{~s}^{-1}$. The objectives of this selection are twofold: first, we are interested in having models representative of High Amplitude $\delta$ Scuti stars (HADS), i.e. with evolutionary stages near the end of the main sequence, and second, the models should have similar global characteristics but for different rotational velocities and metallicities (models $\mathbf{M}_{1 i}$
Table 1. Characteristics of the eight selected $1.8 M_{\odot}$ models with similar evolutionary stages $(\sim 1.3 \mathrm{Gyr})$. From left to right, $\Omega$ is the surface rotational velocity, $T_{\text {eff }}$ the effective temperature (in logarithmic scale), $X_{\mathrm{c}}$ the central hydrogen fraction, $\bar{\rho}$, the mean density, and the last column is the metallicity. $\mathbf{M}_{1 i}$ and $\mathbf{M}_{2 i}$ models have rotational velocities of $\Omega \sim 20$ and $41 \mathrm{~km} \mathrm{~s}^{-1}$, respectively.

\begin{tabular}{cccccc}
\hline \hline Model & $\begin{array}{c}\Omega \\
\left(\mathrm{km} \mathrm{s}^{-1}\right)\end{array}$ & $\begin{array}{c}\log T_{\text {eff }} \\
(\mathrm{K})\end{array}$ & $X_{\mathrm{c}}$ & $\begin{array}{c}\bar{\rho} \\
\left(\mathrm{g} \mathrm{cm}^{-3}\right)\end{array}$ & $\begin{array}{c}{[\mathrm{Fe} / \mathrm{H}]} \\
\mathrm{dex}\end{array}$ \\
\hline $\mathrm{M}_{11}$ & 21.05 & 3.83 & 0.187 & 0.114 & 0.00 \\
$\mathrm{M}_{12}$ & 20.60 & 3.84 & 0.177 & 0.114 & -0.10 \\
$\mathrm{M}_{13}$ & 20.10 & 3.86 & 0.170 & 0.121 & -0.20 \\
$\mathrm{M}_{14}$ & 19.90 & 3.89 & 0.190 & 0.149 & -0.30 \\
\hline $\mathrm{M}_{21}$ & 42.25 & 3.83 & 0.188 & 0.113 & 0.00 \\
$\mathrm{M}_{22}$ & 41.30 & 3.85 & 0.177 & 0.114 & -0.10 \\
$\mathrm{M}_{23}$ & 40.20 & 3.86 & 0.170 & 0.121 & -0.20 \\
$\mathrm{M}_{24}$ & 39.91 & 3.89 & 0.190 & 0.149 & -0.30 \\
\hline
\end{tabular}

and $\mathrm{M}_{2 i}$, with $i=1,4$, of Table 1 , respectively), which permits the study of both effects separately.

\subsection{Mode contamination}

Mode identification is implicit when performing analysis using PD. Indeed, if no additional evidences points towards the observed frequencies as belonging to the corresponding radial mode and the first overtone, the period $\Pi_{1 / 0}$ by itself, does not guarantee such identification. In this context, when rotation is present, the mode contamination plays an important role, since it may change the intrinsic identity of the coupled modes.

It was shown by Daszyńska-Daszkiewicz et al. (2002) that rotational coupling affects the diagnostic diagrams for discriminating the angular degree $\ell$ of a particular mode using multicolour photometry. In particular, the authors found that loci in the amplitude ratio-phase difference diagrams (Garrido 2000) become both aspect and $m$-dependent. In Casas et al. (2006), it was found that mode contamination is significant for radial modes in $\delta$ Scuti stars. Therefore, mode contamination should be then taken into account for the correct identification using amplitude ratio-phase difference diagnostic diagrams. We investigate here how the fundamental radial mode and the first overtone are contaminated by their corresponding $\ell=2, m=0$ coupled pairs.

Coupled modes are described by a linear combination of the involved modes. Following SGM06, for two ${ }^{1}$ degenerate modes, $j$ and $k$, the so called contamination coefficients, $\alpha_{i}(i=j, k)$, defined in Lochard et al. (2007), are written as

$\alpha_{j k}=\frac{\alpha_{j}}{\alpha_{k}}=\frac{m_{j k}^{(1)}+m_{j k}^{(2)}}{m_{j j}^{(1)}+m_{j j}^{(2)} \pm \delta \omega_{0} / 2+\delta \omega_{0}^{2} / 8}$,

where $m_{j k}^{(1)}$ and $m_{j k}^{(2)}$ are the elements of the first- and secondorder correction terms matrices $\mathcal{M}_{j k}$ (SGM06, Eqs. (22) and (23), respectively), and $\delta \omega_{0}=\omega_{0, j}-\omega_{0, k}$. The coefficients are constructed such as the closing relation (normalisation)

$\alpha_{j}^{2}+\alpha_{k}^{2}=1$

is imposed. The coefficients $\alpha_{i}$ are the relative amplitudes of the degenerate modes, and can be interpreted as the relative weight

\footnotetext{
1 The formalism is similar for three or more modes.
} 

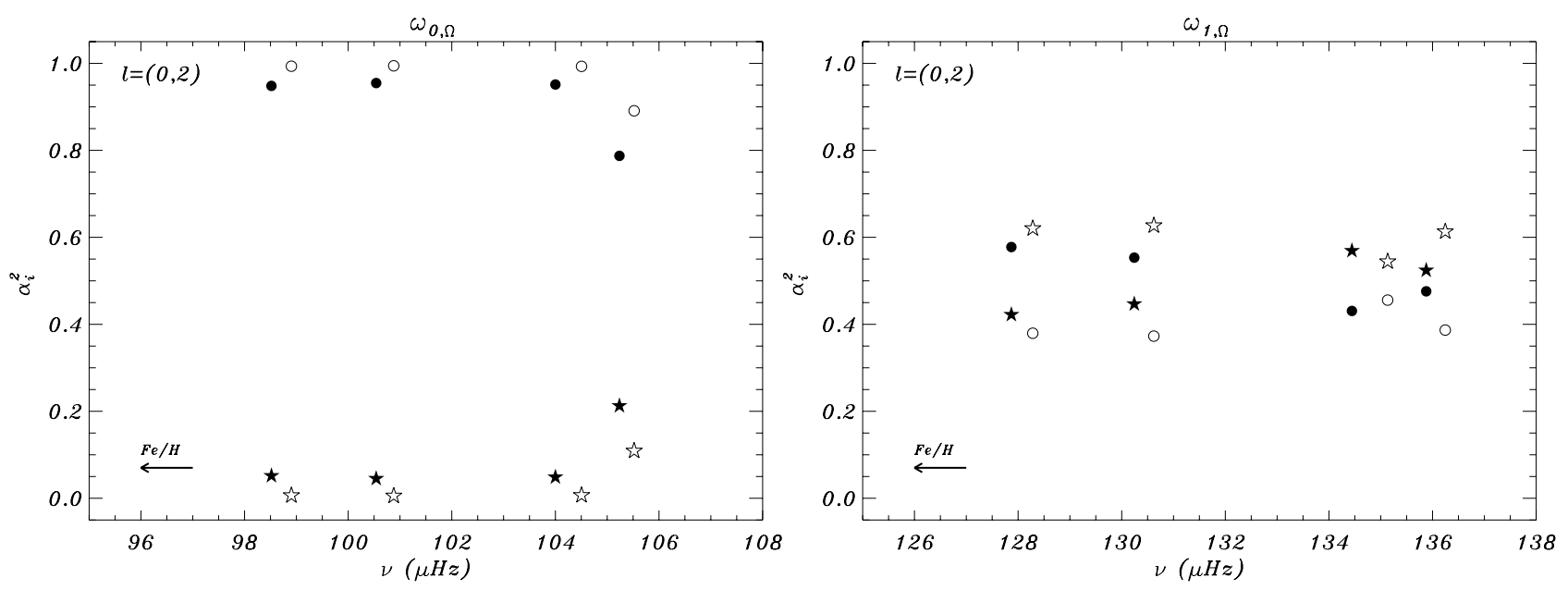

Fig. 1. Contamination coefficients $\alpha_{i}^{2}$ of degenerate pairs $\ell=(0,2)$ as a function of the oscillation frequency $v$ (in $\mu$ Hz) for the selected models listed in Table 1. Left and right panels show $\alpha_{i}^{2}$ for the fundamental radial mode $\omega_{0, \Omega}$ and the first overtone, respectively. As in Fig. 2, filled symbols represent $\alpha_{i}^{2}$ calculated for the selected models with $\Omega \sim 41 \mathrm{~km} \mathrm{~s}^{-1}$, and empty symbols represent $\alpha_{i}^{2}$ calculated for the selected models with $\Omega \sim 20 \mathrm{~km} \mathrm{~s}^{-1}$. Circles and stars represent the radial modes and the corresponding $\ell=2, m=0$ modes, respectively.

of each mode in $\xi$, or otherwise, the degree of contamination of one degenerate mode into another.

For the selected models in Table 1 we computed the relative intrinsic mode amplitudes $\alpha_{i}^{2}$ of the fundamental radial mode $\omega_{0, \Omega}$, the first overtone $\omega_{1, \Omega}$, and their corresponding coupled pairs. The results are shown in Fig. 1, in which the coefficients $\alpha_{i}^{2}$ are depicted as a function of the oscillation frequency of the fundamental radial mode. Similar $\alpha_{i}^{2}$ values are found for the two rotational velocities which confirms the independence of mode contamination upon the rotational velocity. In the case of the fundamental radial mode (left panel), we found that $\alpha_{i}^{2} \sim 1$ for the radial modes and $\alpha_{i}^{2} \sim 0$ for the coupled $\ell=2, m=0$ pairs, except for the lowest metallic model, for which these values are $\alpha_{j} \sim 0.8$ and $\alpha_{k} \sim 0.2$, respectively. This means that both the fundamental radial mode and its coupled pair keep their original identity and can be still regarded as a radial and a quadrupole mode, respectively. On the contrary, the contamination coefficients for the first overtone and its coupled pair (right panel) are of the order of 0.60 and 0.40 , respectively, which implies that these rotationally coupled modes exhibit a mixed radial/quadrupole identity. Notice that, for the model with $\Omega \sim 41 \mathrm{~km} \mathrm{~s}^{-1}$ (filled symbols), $\alpha_{i}^{2} \sim 0.55-0.6$ for the radial modes and $\alpha_{i}^{2} \sim 0.40-0.45$ for their coupled $\ell=2$ pairs. This situation is inverted for the models with $\Omega \sim 20 \mathrm{~km} \mathrm{~s}^{-1}$ (empty symbols). Interestingly, for the model with $[\mathrm{Fe} / \mathrm{H}]=-0.20$ $(v \sim 134 \mu \mathrm{Hz})$ and a rotational velocity of $\Omega \sim 41 \mathrm{~km} \mathrm{~s}^{-1}$, the coefficients are re-inverted. The origin of such permutations is not evident, and is probably related to the other aspect of these results, which are not yet well understood: the different behaviour of the $\alpha_{i}^{2}$ found for the fundamental radial mode and the first radial overtone. Physically, the origin of such a different behaviour probably resides in the nature of their corresponding coupled pairs. Indeed, the radial order of the quadrupole modes coupled with the fundamental radial modes is systematically lower (in the range of $g$ modes) than the radial order of the quadrupole modes coupled with the first radial overtones. This enhances the weight of the vertical and horizontal displacement eigenfunctions of the quadrupole modes in the coupling terms $m_{j k}^{(1)}$ and $m_{j k}^{(2)}$ (Eq. (1)), near the stellar core and surface, respectively. In order to better understand this, a work is currently in progress to analyse in detail the complex dependence of $\alpha_{i}$ coefficients upon the eigenfunctions of the concerned coupled modes, together with variations in the structure of the star due to rotation and evolution.

This result is important, not only because it implies the existence of patterns in the contamination coefficients that may help us to identify properly the affected modes, but also because it may give us relevant information about the nature of the modes. It would be particularly interesting to compare these results with those of the non-perturbative theory (Reese et al. 2006). Together with accurate multicolour photometry, analysis of mode contamination is such a powerful asteroseismic tool because it may help to discriminate, not only the fundamental radial mode from the first overtone, but also to identify the quadrupole modes coupled with them (if they are present in the observed spectrum).

\subsection{Near-degeneracy effects on oscillation frequencies}

In addition to the problem of mode identification, attention is necessarily focused on the effect of near-degeneracy on the oscillation frequencies. As shown by Soufi et al. (1998) and SGM06, such an effect cannot be neglected for asteroseismic studies of moderately rotating stars. As is shown later on in the paper, although HADS are relatively slow-rotating stars, and thereby near-degeneracy effects on the frequencies are expected to be small, they can modify the $\Pi_{1 / 0}(\Omega)$ period ratios sufficiently to cause significant variations, even critical, in the PD. Such an effect is far from trivial and deserves special attention. As shown in SGM06, the impact of near-degeneracy on the oscillation frequencies for $\ell=(0,2)$ degenerate pairs is quite significant for $p$ modes. For such coupled pairs, only second-order coefficients are kept, thus we define the coefficient (SGM06, Eq. (57)):

$C_{j k}^{(2)} \equiv-\omega_{2} \pm \frac{\delta \omega_{0}}{2}$

for near-degenerate modes with $\ell_{j}=\ell_{k} \pm 2$, which represents the second-order frequency corrections for near-degeneracy, and $\omega_{2}$ is the second-order frequency corrections free from neardegeneracy effects. For the sake of brevity, in the following, this coefficient is called coupling strength. We recall that the selection rules for second-order, near-degenerate modes restrict the coupling to modes with $\Delta m=0$, i.e. to modes with the same 

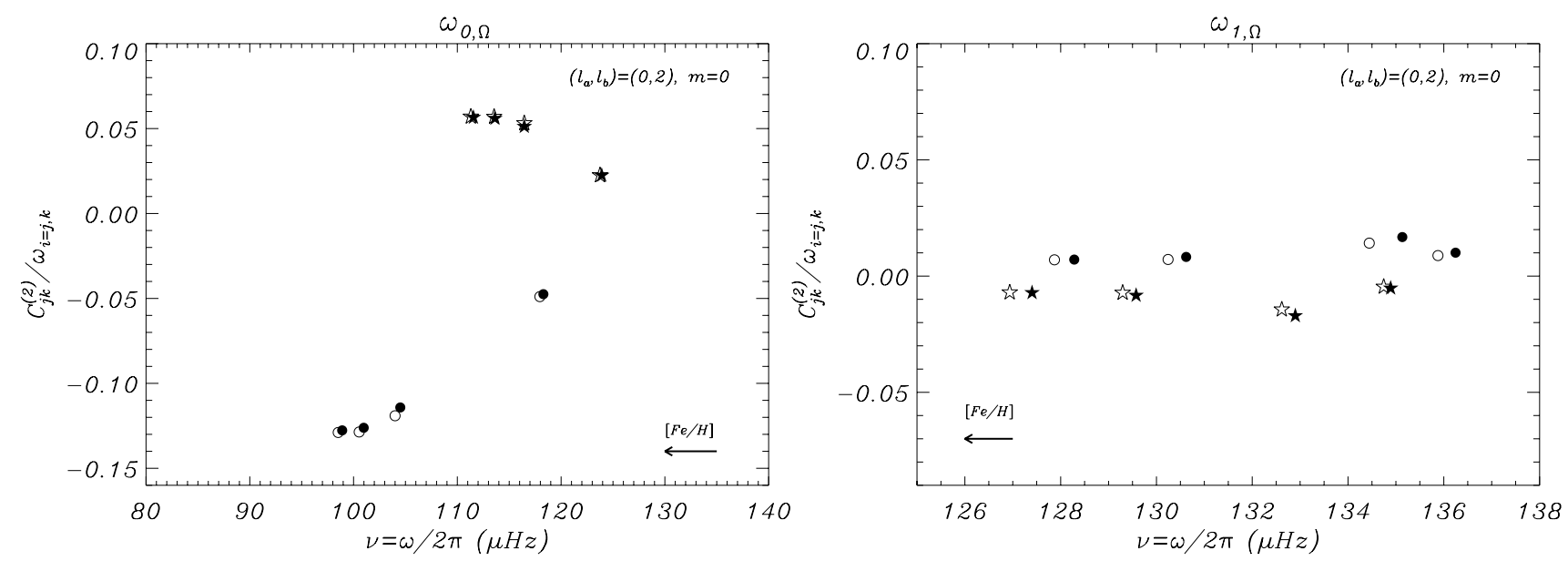

Fig. 2. Coupling strength coefficient normalised to the oscillation frequency as a function of the oscillation frequency $(v=\omega / 2 \pi)$ for degenerate pairs $\left(\ell_{1}, \ell_{2}\right)=(0,2)$ corresponding to the fundamental radial mode (left panel) and the first overtone (right panel). Circles and stars represent the coupling strength coefficients for the radial modes and their corresponding quadrupole coupled modes, respectively. Those calculated for the selected models with $\Omega \sim 41 \mathrm{~km} \mathrm{~s}^{-1}$ are represented with filled symbols, and those calculated for the selected models with $\Omega \sim 20 \mathrm{~km} \mathrm{~s}^{-1}$ are represented by empty symbols.

azimuthal order. This means that radial modes can be coupled only with $(\ell, m)=(2,0)$ modes, which are expected to be less affected by near-degeneracy than for $m \neq 0$ coupled modes (see Sect. 5.5 in SGM06 for more details).

In SGM06 the second-order near-degenerate coefficients $C_{j k}^{(2)}$ for $(\ell, m)=(2,0)$ coupled pairs were studied for a typical $\delta$ Scuti stellar model of $1.8 M_{\odot}$, with solar metallicity. It was found that the coupling strength increases with the rotational velocity. In the case of the fundamental radial mode and the first overtone, which are found typically to be in the range of $50-200 \mu \mathrm{Hz}$ for $\delta$ Scuti stars, the coupling strength $\left|C_{j k}^{(2)}\right|$ can vary from 0.05 to 0.13 , approximately.

As shown in Paper I, the difference in period ratios increases with the rotational velocity for a given metallicity. It is therefore necessary to study the effect of coupling strength on the fundamental radial mode and the first overtone when the rotational velocity and metallicity are varied. For this purpose we use the models of Table 1. In Fig. 2, the normalised coupling strength $C_{j k}^{(2)} / \omega_{j}$ for the fundamental radial mode $\omega_{0, \Omega}$ (left panel) and the first overtone $\omega_{1, \Omega}$ (right panel), together with the coupling strength coefficients of their corresponding coupled quadrupole pairs, are displayed for the selected models. The normalisation of the coefficients to the oscillation frequency is necessary to remove contamination due to the effects of evolution. Notice that the behaviour of the $C_{j k}^{(2)} / \omega_{j}$ for both the fundamental radial mode (left panel) and the first overtone (right panel) is similar for the two rotational velocities. The coupling strength is proportional to the rotational velocity, so logically we expect larger effects for the fastest models. However, the selected rotational velocities are too low to show significant differences. Moreover, the coupling strength of the first radial overtone is independent of the metallicity, showing values of $\left|C_{j k}^{(2)} / \omega_{j}\right| \lesssim 0.03$ for all the selected models. In contrast, the coupling coefficient of the fundamental radial mode shows a clear dependence upon the metallicity, showing absolute values increasing with the metallicity that range from roughly 0.05 to 0.15 . Such values imply variations from 0.005 to $0.1 \mu \mathrm{Hz}$ in the oscillation frequencies, which represent a non-negligible percentage of the total effect of rotation on the oscillation frequencies. We suspect that, as for the contamination coefficients, the influence of the quadrupole coupled modes is mainly the responsible for such different sensitivities of the $C_{j k}^{(2)} / \omega_{j}$ to the metallicity, although other explicit dependencies in the coupling terms, viz, distribution of density and metallicity in the stellar interior, may also play an important role (work in progress).

Furthermore, the effect of near-degeneracy on the oscillation frequencies also depends on the closeness of the mode frequencies (the closer the mode frequencies the larger the coupling strength). Due to this dependence, the coupling strength coefficients are sensitive to the evolutionary stage of the star through the avoided-crossing phenomenon (this is discussed later in Sect. 4). In Paper I (Fig. 3), it was shown that the first radial overtone mode is, in general, more affected by rotation effects (without taking near-degeneracy effects into account) than the fundamental radial mode. On the other hand, when near-degeneracy effects are considered, such a behaviour can be reversed, like in Fig. 2.

This different behaviour is responsible for the wriggles shown in Fig. 3 by both the fundamental radial mode and the first overtone during the evolution (expressed as the logarithm of the period, in days, of the fundamental radial mode). These wriggles, which are visible in both modes, are then transfered to the period ratios. In Fig. 4 we show how, when near-degeneracy is considered, wriggles modify the characteristic smoothed behaviour of RPD. In the case depicted here, wriggles change the period ratios up to 0.02 .

This implies a significant increase of the uncertainty in mass and metallicity determinations using RPD. Nevertheless, for the rotational velocities considered here, the wriggles remain in the range of period ratios typically found for Pop. I stars, [0.772, 0.776], which reduces possible confusion with other radial mode period ratios, and helps to disentangle period ratios of nonradial modes.

\section{The $\Pi_{1 / 0}^{d}(\Omega)$ period ratios and the metallicity/mass determinations}

For the sake of clarity, $\Pi_{1 / 0}^{\mathrm{d}}(\Omega)$ are used when referring to period ratios corrected for near-degeneracy, in contrast to those used in Paper I, $\Pi_{1 / 0}(\Omega)$, which does not include them. In order 

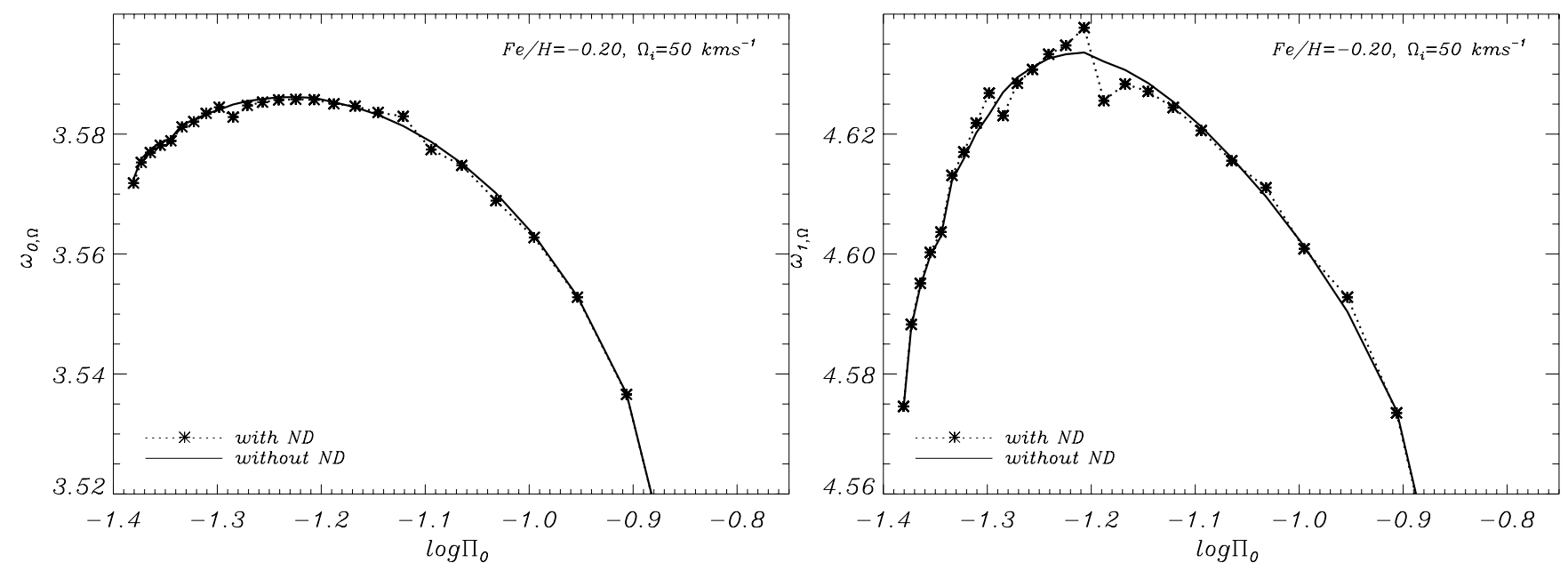

Fig. 3. Fundamental radial mode $\omega_{0, \Omega}$ (left panel) and the first radial overtone $\omega_{1, \Omega}$ (right panel) normalised dimensionless frequencies (to $\sqrt{G M / R^{3}}$ ) as a function of the logarithm of the fundamental radial mode period (in d). Tracks correspond to $1.8 M_{\odot}$ models with $[\mathrm{Fe} / \mathrm{H}]=-0.20$ and an initial rotational velocity of $\Omega_{\mathrm{i}}=50 \mathrm{~km} \mathrm{~s}^{-1}$. Dotted lines with asterisks and solid lines represent frequencies corrected and non-corrected for near-degeneracy effects, respectively.

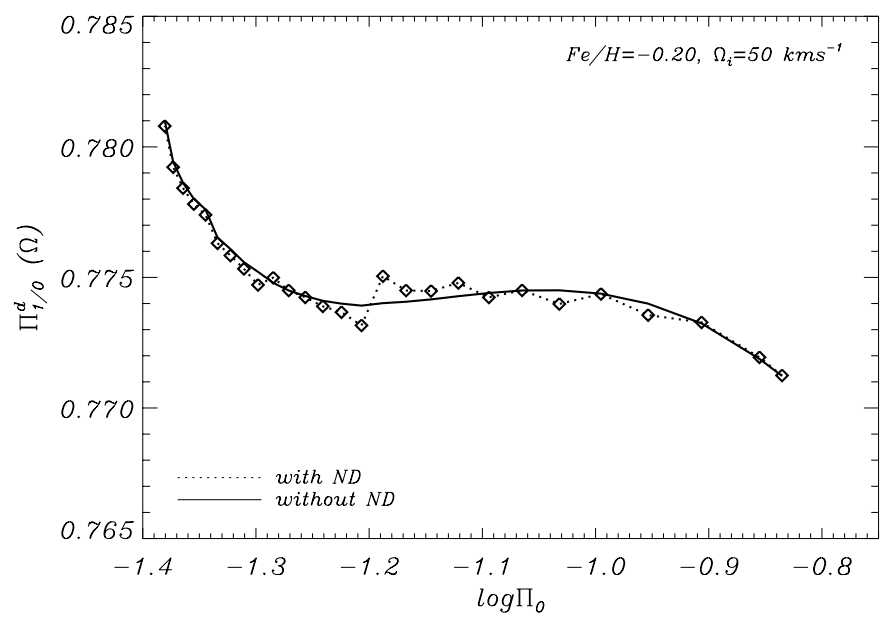

Fig. 4. RPD ( $\Pi_{0}$ in d) illustrating the effect of near-degeneracy on the period ratios. Tracks correspond to $1.8 M_{\odot}$ models with $[\mathrm{Fe} / \mathrm{H}]=$ -0.20 . Wriggles are clearly visible when near-degeneracy (ND) is taken into account.

to determine how near-degeneracy affects metallicity determinations using RPD, we proceed as in Paper I and select, from our model grid, several evolutionary tracks with different metallicities: $[\mathrm{Fe} / \mathrm{H}]=0,-0.1,-0.2,-0.3,-0.35,-0.50$, and $-1.00 \mathrm{dex}$, two different initial rotational velocities $\Omega_{\mathrm{i}}=25,50$, and a fixed mass of $1.8 M_{\odot}$. For each model we then computed the corresponding $\Pi_{1 / 0}^{\mathrm{d}}(\Omega)$ period ratio. In Fig. 5 we show RPD displaying such period ratios, from top to bottom, for tracks computed for $\Omega_{\mathrm{i}}=25$ to $50 \mathrm{~km} \mathrm{~s}^{-1}$, respectively. As expected, the inclusion of the near-degeneracy effects in the frequency corrections does not modify the general behaviour of the period ratios with the metallicity, i.e. $\Pi_{1 / 0}^{\mathrm{d}}(\Omega)$ increases with increasing rotational velocities (left panels). As discussed in Paper I, this is equivalent to a decrease in the metallicity in standard PD. However, the presence of wriggles may inverse this situation in the regions where the curves cross each other. In order to compare quantitatively the evolution of $\Pi_{1 / 0}^{\mathrm{d}}(\Omega)$, with respect to classical non-rotating $\mathrm{PD}$, we use the period ratio differences defined as

$$
\delta \Pi_{1 / 0} \mathrm{~d}(\Omega,[\mathrm{Fe} / \mathrm{H}])=\left[\Pi_{1 / 0}^{\mathrm{d}}(\Omega)-\Pi_{1 / 0}\right]_{[\mathrm{Fe} / \mathrm{H}]} .
$$

For the lowest rotational velocities $\left(\Omega_{\mathrm{i}} \leq 25 \mathrm{~km} \mathrm{~s}^{-1}\right)$, the size of wriggles is almost negligible (Fig. 5, top panels), except for certain models; thus the discussion on the effect of rotation on period ratios given in Paper I remains valid, and the effect of neardegeneracy in terms of period ratios differences is $\delta \Pi_{1 / 0}{ }^{\mathrm{d}} \sim 2-$ $3 \times 10^{-3}$. For higher rotational velocities (see Fig. 5, bottom panels), wriggles are large which imply period ratios differences of $\delta \Pi_{1 / 0}{ }^{\mathrm{d}} \sim 10^{-3}$ for some models, and close to $10^{-2}$ for others. The largest differences are found for main-sequence models, but significant differences are also observed for more evolved evolutionary stages.

Let us now analyse the effect of near-degeneracy on RPD in terms of metallicity. For shortness, we adopt the same nomenclature used in Paper I to identify the different tracks as a function of the metallicity and rotational velocity. For instance, we use $[-0.1]_{25}$ when referring to models with $\Omega_{\mathrm{i}}=25 \mathrm{~km} \mathrm{~s}^{-1}$ and $[\mathrm{Fe} / \mathrm{H}]=-0.1$. For the lowest initial rotational velocity considered, $\Omega_{\mathrm{i}}=25 \mathrm{~km} \mathrm{~s}^{-1}$, the results are similar to those obtained without considering near-degeneracy effects. This means that, even considering the wriggles in the period ratios, the confusion between tracks remains around $0.10-15$ dex. More important is the case of models with $\Omega_{\mathrm{i}}=50 \mathrm{~km} \mathrm{~s}^{-1}$, for which large wriggles are responsible for significant uncertainties in metallicity determinations. In particular, rotating tracks $[0.00]_{50}$ may be confused with $[-0.35]_{0}$ tracks, and $[-0.10]_{50}$ may be confused with non-rotating $[-0.50]_{0}$ tracks. This implies that the uncertainty of metallicity determinations may reach $0.50 \mathrm{dex}$, which is critical for Pop. I HADS. Analysis of the period ratios in terms of mass yields similar results to those obtained in Paper I, i.e. $\Pi_{1 / 0}^{\mathrm{d}}(\Omega)$ increases with increase in mass for any rotating track. In Fig. 6, we compare rotating and non-rotating tracks for different masses. In particular, it can be seen that the period ratios for $1.8 M_{\odot}$ models with $\Omega_{\mathrm{i}}=50 \mathrm{~km} \mathrm{~s}^{-1}$ and $[\mathrm{Fe} / \mathrm{H}]=-0.20$ can be confused with $2.30 M_{\odot}$ non-rotating models with solar metallicity. This yields an uncertainty of $0.5 M_{\odot}$, which cannot be neglected in any mass determination through PD. A detailed analysis of the uncertainty on period ratios caused by rotation effects (including near-degeneracy), as well as by the mass and other physical parameters (namely the overshooting and mixinglength parameter), is currently in preparation. 

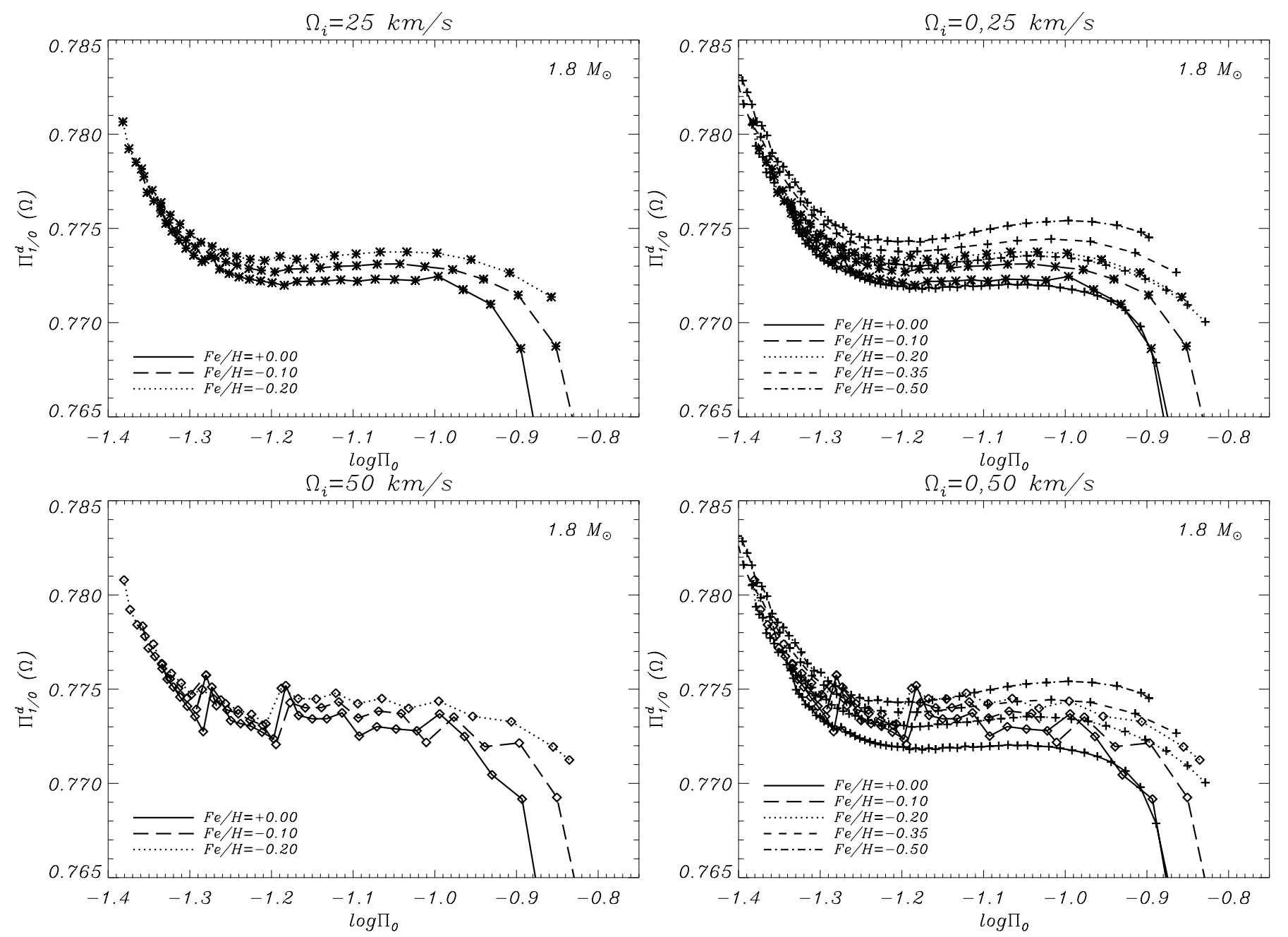

Fig. 5. Theoretical RPD including near-degeneracy effects on frequencies. The $\Pi_{1 / 0}^{\mathrm{d}}(\Omega)$ period ratios have been computed in the manner described in Sect. 2 for a set of evolutionary $1.8 M_{\odot}$ tracks obtained for different metallicities. Tracks for two initial rotational velocities are considered: 25 and $50 \mathrm{~km} \mathrm{~s}^{-1}$ (from top to bottom). Left panels show only rotating models. Right panels show the comparison of rotating and non-rotating tracks (classic PD). For convenience, the following symbols are used: crosses, representing non-rotating models; asterisks, representing models evolved with $\Omega_{\mathrm{i}}=25 \mathrm{~km} \mathrm{~s}^{-1}$; diamonds and those evolved with $\Omega_{\mathrm{i}}=50 \mathrm{~km} \mathrm{~s}^{-1}$.

In general, near-degeneracy effects complicate the already confusing scenario stated in Paper I, in which near-degeneracy was not considered. Nevertheless, such an apparent worsening of the situation, may in fact be a source of information, provided that wriggles in PD are not placed randomly. Indeed, rotational coupling is sensitive to the so-called avoided-crossing phenomenon, i.e. the well-known phenomenon occurring when the convective core recedes during the main sequence evolution. The relation between near-degeneracy and the avoided-crossing phenomenon comes from the nature of the near-degeneracy itself, which takes place when two or more modes close in frequency fulfil certain selection rules (DG92, SGD98, SGM06). These selection rules restrict the mode coupling to modes having the same azimuthal order $m$ and $\Delta \ell=0, \pm 2$. The case treated here concerns $\ell=0$ and $(\ell=2, m=0)$ modes, which are naturally close in frequency, and then systematically coupled. This can be easily seen in Fig. 7, which shows the evolution of the radial order values of the quadrupole pairs $\left(n_{0, \mathrm{c}}\right.$ and $\left.n_{1, \mathrm{c}}\right)$ with which the fundamental radial mode and the first overtone are coupled. During the evolution, the pairs $\left(n_{0, \mathrm{c}}, n_{1, \mathrm{c}}\right)$ decrease their values following a given pattern, which is clearly modulated by the aforementioned avoided-crossing phenomenon. For the radial modes, such a modulation is different from the modulation occurring in the corresponding quadrupole coupled modes. That is, the step features shown in Fig. 7 occurs for $n_{0, \mathrm{c}}$ at different evolutionary stage than for $n_{1, \mathrm{c}}$, which causes the wriggles in the period ratios. This can be verified by comparing Fig. 7 with Fig. 3 for modes around $\log \Pi_{0} \sim[-1.3,-1.2]$.

Interestingly, the results shown in Fig. 7 are nearly independent of the metallicity and the rotational velocity ${ }^{2}$, and present some slight dependence upon the mass. This may be helpful to identify the quadrupole modes coupled with the radial modes, together with other mode identification techniques. For such cases, with accurate determinations of metallicity provided, analysis of this phenomenon may also be able to help in constraining the mass of the models.

\section{Conclusions}

The effect of near-degeneracy in RPD has been analysed. Following Paper I, detailed seismic models have been computed considering rotation effects on both equilibrium models and on adiabatic oscillation frequencies. A grid of $1.8 M_{\odot}$ stellar equilibrium models, similar to the one used in Paper I (completed

\footnotetext{
${ }^{2}$ In the range of rotational velocities considered in this work.
} 


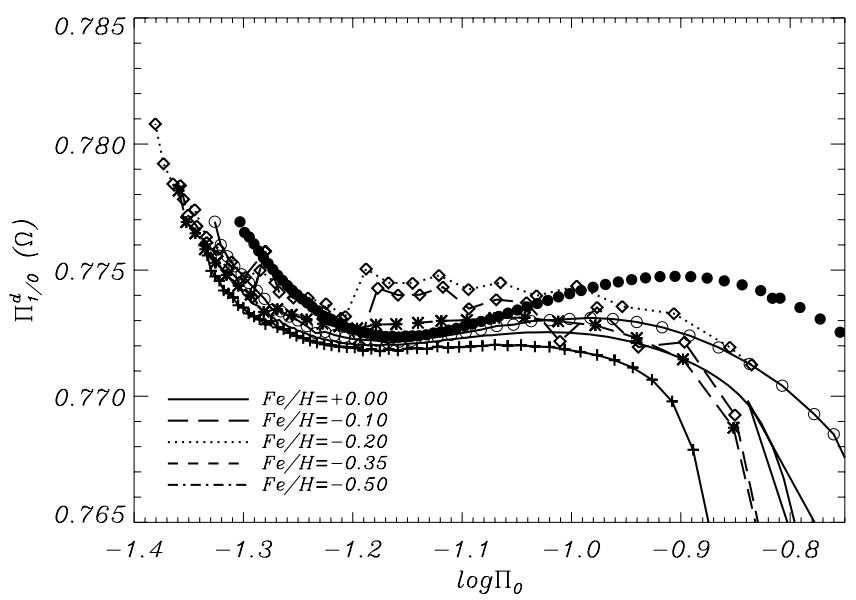

Fig. 6. RPD ( $\Pi_{0}$ in d) illustrating the effect of considering different masses. Symbols have the same meaning of those from Fig. 5, except for empty and filled circles, which correspond to 2.00 and $2.30 M_{\odot}$ nonrotating models and solar metallicity, respectively.

with $[\mathrm{Fe} / \mathrm{H}]=-0.30$ models), has been used. From these models, the corresponding oscillation eigenfrequencies corrected for the effect of rotation up to the second order, including neardegeneracy effects, have been computed.

Near-degeneracy effects have been divided into two components: the effect of mode contamination and the coupling strength. The former effect concerns the relative spherical harmonic amplitude of degenerate modes. Analysis of such relative amplitudes (contamination coefficients) reveals that the identity of the degenerate fundamental radial mode remains almost unaltered, while the degenerate first overtone presents a mixed radial/quadrupole identity. This must then be taken into account when performing $\ell$-diagnostics using amplitude ratio/phase difference diagrams from multicolour photometry. Indeed, analysis of mode contamination enhances such diagnostic diagrams since not only may it help to discriminate the fundamental radial mode from the first overtone, but also to identify the quadrupole modes coupled with them (if they are present in the observed spectrum).

The effect of near-degeneracy on the oscillation frequencies (coupling strength) has also been studied. Although this effect increases for increasing rotational velocities, for the rotational velocities considered here, similar values of the couple strength coefficients are found when changing the rotational velocity of the models. In terms of frequency variations, the coupling strength analysis suggests that near-degeneracy may modify the frequency of the fundamental radial mode and the first overtone up to $0.3 \mu \mathrm{Hz}$, which represents a non-negligible percentage of the total effect of rotation on the oscillation frequencies. Moreover, near-degeneracy affects the frequency of the fundamental radial mode and the first overtone differently. In particular, the largest effect on the frequencies is found alternatively for the fundamental radial mode and the first overtone, depending on the evolutionary stage. This alternation is responsible for the wriggles shown in the fundamental-to-first overtone period ratios when near-degeneracy effects are taken into account. Analysis of these wriggles in the tracks, which are larger for increasing rotational velocities, reveals that they become significant (in the context of RPD) and degrade substantially the accuracy of period ratios $\Pi_{1 / 0}^{\mathrm{d}}(\Omega)$, which can reach up $10^{-2}$. In terms of metallicity, this implies uncertainties reaching up to $0.50 \mathrm{dex}$ (for the largest rotational velocity considered), which is critical for Pop. I HADS. Furthermore, near-degeneracy

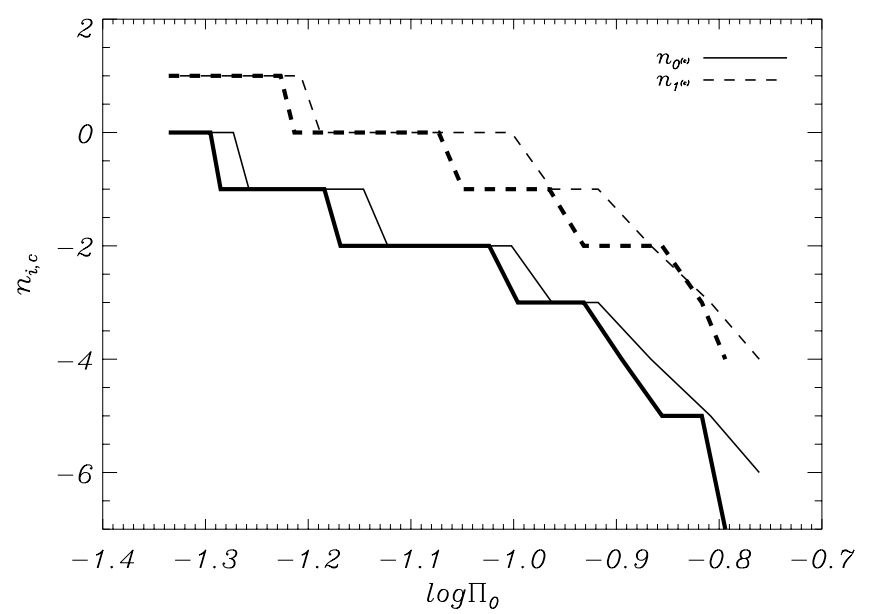

Fig. 7. Radial order $n_{0,(\mathrm{c})}$ and $n_{1,(\mathrm{c})}$ of the $\ell=2, m=0$ pairs respectively coupled with the fundamental radial mode and the first overtone, as a function of the logarithm of the fundamental radial mode period (in days). Thick lines correspond to models of $1.8 M_{\odot}$, and thin lines to models with $2 M_{\odot}$, both computed with $\Omega_{\mathrm{i}}=25 \mathrm{~km} \mathrm{~s}^{-1}$ and solar metallicity.

effects also increase the uncertainty in mass determination using RPD, which can reach $0.5 M_{\odot}$ for certain models. In general, for stellar rotational velocities larger than $15-20 \mathrm{~km} \mathrm{~s}^{-1}$, this new scenario would thus invalidate, a priori, the PD as diagnostic diagrams. However, it is found that wriggles in period ratios are not located randomly in PD. They depend on the frequency evolution of the quadrupole modes coupled with the radial ones, which are mainly modulated by the avoided-crossing phenomenon. Moreover, it is found that such a behaviour is dependent on the mass of the star, but is nearly independent of the rotational velocity and metallicity. These results provide clues for the mode identification of the fundamental radial mode, the first overtone, and their corresponding quadrupole coupled modes, using only white light photometry. If additional information from multicolour photometry and/or spectroscopy is given, RPD may be a powerful asteroseismic technique not only for diagnostics on metallicity and/or mass, but also for the rotational velocity (and thereby the inclination angle).

Acknowledgements. This study would not have been possible without the financial support of the Instituto de Astrofísica de Andalucía (CSIC) by an I3P contract financed by the European Social Fund and from the Spanish "Plan Nacional del Espacio" under project ESP2004-03855-C03-01.

\section{References}

Casas, R., Suárez, J. C., Moya, A., \& Garrido, R. 2006, A\&A, 455, 1019 Daszyńska-Daszkiewicz, J., Dziembowski, W. A., Pamyatnykh, A. A., \& Goupil, M.-J. 2002, A\&A, 392, 151

Dziembowski, W. A., \& Goode, P. R. 1992, ApJ, 394, 670

Garrido, R. 2000, in Delta Scuti and Related Stars, Reference Handbook and Proceedings of the 6th Vienna Workshop in Astrophysics, held in Vienna, Austria, 4-7 August, 1999, ed. M. Breger, \& M. Montgomery (San Francisco: ASP), ASP Conf. Ser., 210, 67

Lochard, J., Suárez, \& Goupil, M. J. 2007, in preparation

Morel, P. 1997, A\&AS, 124, 597

Pamyatnykh, A. A. 2003, Ap\&SS, 284, 97

Reese, D., Lignières, F., \& Rieutord, M. 2006, A\&A, 455, 621

Soufi, F., Goupil, M. J., \& Dziembowski, W. A. 1998, A\&A, 334, 911

Suárez, J. C. 2002, Ph.D. Thesis, ISBN 84-689-3851-3, ID 02/PA07/7178

Suárez, J. C., Garrido, R., \& Goupil, M. J. 2006a, A\&A, 447, 649 (Paper I)

Suárez, J. C., Goupil, M. J., \& Morel, P. 2006b, A\&A, 449, 673 (SGM06)

Tran Minh, F., \& Léon, L. 1995, in Physical Processes in Astrophysics. ed. I. W.

Roxburg, J. L. Maxnou, (Springer Verlag, Berlin), 219 